\title{
Triage of colonoscopies: open access endoscopy versus outpatient consultation with a gastroenterologist
}

Authors

Institution
C.D. Wichers, N.C.M. van Heel, F. ter Borg, M.A. van Herwaarden

Gastroenterology, Deventer Hospital, Deventer, the Netherlands submitted 11. April 2014 accepted after revision 5. May 2014

\section{Bibliography}

Dol http://dx.doi.org/ 10.1055/s-0034-1377325 Published online: 17.9.2014 Endoscopy International Open 2014; 2: E187-E190 (c) Georg Thieme Verlag KG Stuttgart · New York E-ISSN 2196-9736

\section{Corresponding author} Carmen D. Wichers Deventer HospitalGastroenterology Nico Bolkestaeinlaan 75 Deventer 7416 SE Netherlands Fax: +31612872053 cdwichers@hotmail.com
Background and study aims: In many Dutch hospitals, open access referral for colonoscopy is authorized by a gastroenterologist after screening a standard referral letter (SRL) without face-to-face contact with the patient. We investigated the added value of a $7.5 \mathrm{~min}$ outpatient consultation with a gastroenterologist $(\mathrm{OC})$, regarding the patient indications, priority for colonoscopy, and the frequency of correct information about patient medications and comorbidities on SRLs.

Patients and methods: In a prospective, blinded, single-center study, gastroenterologists assessed SRLs for the accuracy and priority of the colonoscopy request (SRL). These data were compared to results from the OC, and primary outcomes were the number of patients who were not recommended for colonoscopy and priority scheduling of colonoscopy for suspicion of cancer.

Results: Patients were analyzed using both SRL and OC and, of 255 patients, 224 of them underwent colonoscopy. Colonoscopy was not recommended for $6.3 \%$ and $11.4 \%$ of patients using the

\section{Introduction}

$\nabla$

Colonoscopy is considered the "gold standard" for colorectal cancer screening [1]. A diagnostic colonoscopy can be performed safely without prior consultation with a gastroenterologist, which increases efficiency and reduces delays and costs $[2,3,4]$. This practice of "open access endoscopy" (OAE) has gained popularity, particularly in the Western world. Despite these benefits, concerns exist about improper referrals or patients referred for colonoscopy in whom the possible risks outweigh the benefits such as patients with serious comorbidities, those who are frail, and those with a limited life expectancy. In addition, improper referrals for colonoscopy may increase complications, average waiting time for services, and health care costs [5].
SRL and OC, respectively $(P=0.02)$. Using the SRL, gastroenterologists did not recommend colonoscopy for seven patients, but the same patients were recommended for colonoscopy when OC was available. This was explained because the indications on the SRL did not match the information obtained from OC. Compared to OC, more colonoscopies were prioritized when the SRL was used to make decisions. Cancer was detected in 7/112 (SRL ) versus 7/65 (OC ) of priority-scheduled patients. SRLs did not report the use of coumarins and insulin in $1.6 \%$ of patients or the prevalence of serious comorbid conditions in $52 \%$ of patients.

Conclusions: A $7.5 \mathrm{~min}$ outpatient consultation with a gastroenterologist improved the identification of indications for colonoscopy, decreased priority scheduling of patients, and increased the number of patients diagnosed with cancer in the prioritized group. SRLs frequently omitted patients' medications and comorbidities.

In many centers, standardized referral letters (SRL) are screened by gastroenterologists to evaluate whether patients should be referred for colonoscopy, medications, and comorbidities. Unfortunately, SRLs do not always provide accurate information; therefore, we introduced a $7.5 \mathrm{~min}$ outpatient consultation (OC), performed by a gastroenterologist, to ascertain key features about patients to determine if colonoscopy is warranted before authorizing the procedure. In this study, we prospectively evaluated the SRL and the OC to define the differences in outcomes regarding regarding recommendations for or against colonoscopy and patient priority scheduling. In addition, the patients' use of medications (in particular, insulin and coumarin) and comorbidities were recorded. 


\section{Patients and methods}

This prospective, observational, single-center study was performed at the Department of Gastroenterology, Deventer Hospital, The Netherlands. Formal ethics committee review or approval was not required. Between May 2012 and July 2012, all consecutive patients referred for surveillance and diagnostic colonoscopy were de-identified and included in the analysis. There were no exclusion criteria. Two methods of identifying patients for colonoscopy were evaluated: the standardized referral letter (SRL) and the outpatient consultation (OC).

Standardized referral letter: The SRL is a predefined list of approved indications and symptoms that warrant colonoscopy. This includes patients' use of anticoagulants, a diagnosis of diabetes, with or without insulin therapy, and contagious diseases. Current medications and serious comorbidities were also included. All SRLs were scrutinized by an independent gastroenterologist who was instructed to use only the SRL for authorization of colonoscopies. In instances where authorization for a colonoscopy was approved, the gastroenterologist determined the patient's priority for undergoing the colonsocopy: A, within one week; B, within two weeks; and $C$, first regular opportunity (usually within 3-4 weeks). Gastroenterologists were selected using a day-by-day rotation system, ensuring that they had no previous contact with the study patients or their medical records.

Outpatient consultation: Every outpatient referred for colonoscopy had a standard 7.5 min face-to-face consultation with a gastroenterologist who verified the data in the patient's SRL. The outpatient consultation occurred before and in addition to colonoscopy, but without a physical examination. Additional information regarding patient symptoms, comorbidities, and use of medications was obtained from the patient and the electronic medical record system. The gastroenterologist subsequently approved or did not approve the patient for colonoscopy. When a colonoscopy was authorized, a priority level was given for that patient, and the patient met with a nurse for additional information about the procedure. All patients gave informed consent prior to the procedure. When colonoscopies were not authorized for a patient, both the patient and the referring physician were informed by the gastroenterologist that the patient did not meet the criteria for the procedure. Colonoscopies were performed by experienced endoscopists, including five gastroenterologists and three nurse endoscopists. All procedures were performed under conscious sedation using midazolam. The outcomes of colonoscopies were retrieved from electronic hospital records.

\section{Outcome parameters}

The primary outcome was the number of patients who were not authorized for colonoscopy. Secondary outcomes were the differences in the patient's priority for colonoscopy, the number of malignancies identified, the number of advanced neoplastic lesions identified, and the number of new diagnoses of inflammatory bowel disease (IBD). In addition, the completeness of the SRL with respect to the patient's use of medications (insulin and coumarins) and their important comorbidities were recorded. Important comorbidities were defined as previous abdominal surgery, cardiac disease, chronic obstructive pulmonary disease, malignancies, and coagulation disorders.

\section{Statistical analysis}

The number of patients in this study (255) was determined by the following calculation (because no data were available in the literature): power of $90 \%, \alpha<0.05$, and an estimation of $3 \%$ of pa-

\begin{tabular}{|lclc|}
\hline Table 1 & \multicolumn{3}{c|}{ Results of SRL and OC authorizations for colonoscopy. } \\
& $\begin{array}{l}\text { OC- } \\
\text { authorized }\end{array}$ & $\begin{array}{l}\text { OC-not } \\
\text { authorized }\end{array}$ & Total \\
\hline SRL-authorized & 219 & $20^{1}$ & 239 \\
SRL-not authorized & 7 & $9^{1}$ & $16(6.3 \%)$ \\
\hline Total & 226 & $29(11.4 \%)$ & 255 \\
\hline
\end{tabular}

Abbreviations: $\mathrm{OC}=7.5 \mathrm{~min}$ outpatient consultation; $S R L=$ standard referral letter.

${ }^{1}$ These patients did not have a colonoscopy

tients not authorized for colonoscopy using SRL and 10\% of patients not authorized for colonoscopy using OC. Most of the data in both groups were descriptive. Repeated measurements on a single sample were performed (SRL and OC). McNemar's test was used for nominal data, which is suitable for matched data pairs. For categorical data, the Wilcoxon signed-rank test was used, a $P$-value less than 0.05 was considered to be statistically significant. Statistical analyses were conducted using SPSS 20.0 software.

\section{Results}

$\nabla$

Consecutive patients (255; 51\% men; mean age, 61 years; range, 19-88 years) were included in the study. Eighty-three percent of the patients were referred by a general practitioner, the others were referred by internists and surgeons. After OC, 226 patients (89\%) were authorized for colonoscopy; two patients cancelled their colonoscopies, and 224 colonoscopies were performed (87\%). In 211 (94\%) patients, the colonoscopy extended to the cecum. After SRL, 239 (93.7\%) consecutive patients were authorized for colonoscopy. The results of the SRL and OC authorizations are shown in $\bullet$ Table 1. Fewer colonoscopies were authorized by OC (11.4\% of patients were not authorized for a colonoscopy) than by SRL (6.3\% of patients were not authorized for a colonoscopy; $P=0.02$ ).

As shown in $\bullet$ Table 1, seven of sixteen patients that were not authorized for colonscopy using the SRL, were authorized for colonoscopy using OC, because of incomplete information on the SRL (eg., missing indications). For example, one patient underwent a colonoscopy a year previously, which was sufficient reason to decline the colonoscopy using the information in the SRL. Using OC, however, it was noticed that the prior colonoscopy of this patient was incomplete because of fecal contamination, and the colonoscopy for this patient was then authorized. In five of the seven patients, lesions were found during colonoscopy. These lesions were symptom-related and included hemorrhoids, colonic diverticular disease, and adenomatous polyps.

- Table 2 shows the reasons why colonoscopies were not authorized, which included symptoms related to irritable bowel syndrome (using the Rome-III-criteria), recently performed colonoscopy, incorrect surveillance (early referral or no reason for colonoscopy according to the guidelines), or a patient's choice for a second consultation or diagnostic tests (upper endoscopy, sigmoidoscopy, laboratory tests, or imaging) instead of colonoscopy. Other reasons why colonoscopies were not authorized included changes in patients' medications and referrals to other specialists. More colonoscopies were prioritized when gastroenterologists use the SRL (30\% level A, 14\% level B) than when they used the OC (18\% level A, $8 \%$ level B). Wilcoxon signed-rank test revealed a significant difference in priority assignments $(P<$ $0.001)$. Colorectal cancer was detected in $5.8 \%(13 / 224)$ of pa- 


\begin{tabular}{llll} 
& $\begin{array}{l}\text { SRL } \\
\mathbf{n}(\%)\end{array}$ & $\begin{array}{l}\text { OC } \\
\mathbf{n}(\%)\end{array}$ & P-value \\
\hline $\begin{array}{l}\text { Total number of patients who were not referred for } \\
\text { colonoscopy: }\end{array}$ & $16(6.3 \%)$ & $29(11.4 \%)$ & $0.02^{1}$ \\
\hline $\begin{array}{l}\text { Reasons colonoscopy was not recommended: } \\
\text { Symptoms of IBS (Rome III) }\end{array}$ & $2(12.5 \%)$ & $5(17.2 \%)$ & \\
Recently performed colonoscopy & $5(31.3 \%)$ & $2(6.9 \%)$ \\
$\begin{array}{l}\text { Incorrect surveillance } \\
\text { Second consultation or diagnostic tests }\end{array}$ & $2(12.5 \%)$ & $7(24.1 \%)$ & \\
Other & $8(50.0 \%)$ & $16(55.2 \%)$ & \\
\hline
\end{tabular}

Table 2 Reasons for de-authorization of colonoscopy.

Reasons for colonoscopy was not recommended. Data from 16 (SRL) and 29 (OC) subjects who were not recommended for colonsocopy. Some of the patients were not recommended for more than one reason, causing the total number of reasons that patients were not recommended for colonoscopy (SRL, $\mathrm{n}=18$ and OC, $\mathrm{n}=30$ ) to be higher than the total number of patients who were not recommended for colonoscopy (SRL, $n=16$ and OC, $n=29$ ).Abbreviations: SRL = standard referral letter screening; $O C=7,5$ min outpatient consultation; $\mathrm{BD}=$ inflammatory bowel disease

${ }^{1}$ significance $(P<0.05)$

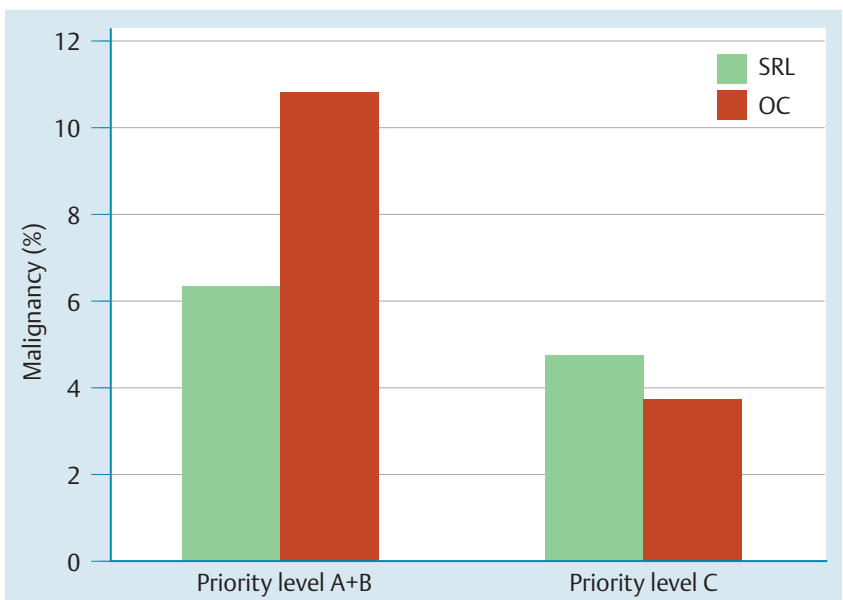

Fig. 1 The assessment of the priority of outpatient colonoscopy based on SRL and OC plotted against the number of malignancies found at colonoscopy. Abbreviations: $\mathrm{SRL}=$ standard referral letter; $\mathrm{OC}=7.5 \mathrm{~min}$ outpatient consultation.

tients who underwent a colonoscopy. These malignancies were confirmed by histopathology of the biopsies that were taken during colonoscopy. Fig. 1 shows that although fewer colonoscopies were prioritized using OC (level $A+B)$, more patients were diagnosed with malignancies $(10.8 \%$ versus $6.3 \%, P<0.001)$. When colonoscopies were not prioritized, malignancies were detected in $4.7 \%$ and $3.7 \%$ of patients using SRL and OC, respectively. The accuracy of prioritization was demonstrated in that the number of patients in level A and B decreased while the number of patients with colorectal cancer in those levels increased. Information about current medications was incomplete in 31.5\% of the SRLs. The most dangerous medications (insulin, coumarins, and platelet inhibitors) were reported in $1.6 \%$ of the SRLs. Medications that patients take are less relevant for colonoscopy than for other procedures, but antihypertensives, beta-blockers, and proton pump inhibitors were missing on the SRLs. Of all patients, $63.5 \%$ had a past medical history, which included abdominal surgery $(35.8 \%)$ cardiac disease $(15.4 \%)$, COPD (3.1\%), prior malignancy (3.7\%), diabetes mellitus (3.0\%), and coagulation disorder (0.6\%). Relevant past medical history was incomplete for $54.9 \%$ of all patients.

\section{Discussion}

In the Netherlands, 191,339 colonoscopies were performed in 2009 [6]. In the current study, SRL, which is current practice, revealed that 16 patients $(6.3 \%)$ did not require colonoscopy whereas OC revealed that 29 patients $(11.4 \%)$ did not require colonoscopy. In the Netherlands, 191,339 colonoscopies were performed in 2009 [6]. Extrapolating the results from this study to the 2009 data revealed that SLR and OC would result in 12,058 and 21,812 fewer annual colonoscopies nationwide, respectively. One could argue that in real practice SRL will result in more unwarranted colonoscopies. After the introduction of the screening program for colorectal cancer (CRC) in the Netherlands, the benefits of the $\mathrm{OC}$ will increase, because the number of patients authorized for colonoscopies will increase by 66,000 annually in the long term [7]. Reducing the number of non-screening colonoscopies by using $\mathrm{OC}$ reduces the workload and the financial consequences.

Nearly half of of those who were not recommended for colonoscopy by gastroenterologists using SRL alone were authorized for colonoscopy using OC, and many of these patients had lesions that were identified during colonoscopy. Thus, there are two problems with SRL; first, fewer patients were authorized to undergo colonoscopy, and, second, patients who were not originally authorized by SRL were ultimately diagnosed with a lesion when the procedure was authorized after OC. This supports the importance of OC for maintaining high quality and safety for patients. Relying on SRL could cause delays in diagnoses of diseases and increase health care costs.

We found OC is better than SRL for establishing patient priority for colonoscopy and that fewer referrals were assessed as urgent (level A or B). Although the number of urgent patient referrals was lower for $\mathrm{OC}$, the number of diagnoses of malignancies was comparable to SRL. This is supported by Ramsay et al., who reported high numbers of urgent patient referrals by general practioners and that in $92.2 \%$ of these no malignancies were found [8]. Determining patient priority is important because of its logistical consequences. A long patient waiting period for a colonoscopy is common for most hospitals. When the number of referrals assessed as urgent is too high, it could cause problems scheduling colonoscopies that actually are urgent.

We found incomplete referral information regarding the patients' past medical history and current medications. In particular, previous abdominal surgery was not reported and it is a factor known to contribute to the difficulty of a colonoscopy $[9,10]$. In 2006, an American study reported inaccurate relevant informa- 
tion in $8.8 \%$ of patient referrals in an assessment of the SRL [11]. In the current study, the incomplete information on the SRLs was revealed upon OCs and the colonoscopies were subsequently approved.

We performed a prospective study, with blinded evaluation of SRL and OC to determine if patients would be recommended for colonoscopy or not. Patients who were not recommended for colonoscopy by OC, did not receive colonoscopy, which is a limitation of this study. It is impossible to evaluate whether these subjects needed a colonoscopy or not. However, that determination was not the purpose of this study, and the number of patients in this group was small relative to the total number of patients. Furthermore, these patients were evaluated by an experienced gastroenterologist who concurred with the decision made by OC. Prioritization of colonoscopies at the Deventer Hospital may be relatively easy because of a short waiting period and this could have influenced the percentages of prioritized patients in our study. However, both SRL and OC were evaluated using the same conditions so the differences noted remain statistically valid. Patients recommended for a colonoscopy using OC met with a nurse for additional procedural information.This has a positive impact on the quality of bowel preparation, however, the impact of this meeting was not evaluated in this study.

The study shows that the implementation of OC to authorize pateint referrals for colonoscopy reduced the number of authorizations for patients with improper indications. Furthermore, it reduced the number of procedures that were not authorized from the SRL, provided a better prioritization of patients for colonoscopies, and provided important additional information that was lacking on the SRL. These findings show that OC provides important health care benefits for patients referred for colonoscopies, a reduced of risk of colonoscopies that are not justified or among patients that are too frail, and reducted costs and patient waiting times.

\section{Abbreviations

\section{Competing interests: None}

\section{References}

1 Brenna E, Skreden K, Waldum HL. The Benefit of Colonoscopy. Scand J Gastroenterol 1990; 25: 81-88

2 Charles RJ, Cooper GS, Wong RC et al. Effectiveness of open-access endoscopy in routine primary-care practice. Gastrointest Endosc 2003; 57 : $183-186$

3 Charles RJ, Chak A, Cooper GS et al. Use of open access in GI endoscopy at an academic medical center. Gastrointest Endosc 1999; 50: 480485

4 Mahajan RJ, Marshall JB. Prevalence of open-access gastrointestinal endoscopy in the United States. Gastrointest Endosc 1997; 46: 21-26

5 Zuccaro GJr, Provencher K. Does an open access system properly utilize endoscopic resources? Gastrointest Endosc 1997; 46: 15-20

6 van Turenhout ST, Terhaar siveDroste JS, Meijer GA et al. Anticipating implementation of colorectal cancer screening in The Netherlands: a nationwide survey on endoscopic supply and demand. BMC Cancer 2012; 46: DOI 10.1186/1471-2407-12-46

7 Rijksinstituut voor Volksgezondheid en Milieu (RIVM). Feasibility study into population screening for bowel cancer. Detection of bowel cancer put into practice. 2011: 225082001

8 Ramsay G, MacKay C, Nanthakumaran S et al. Urgency of referral and its impact on outcome in patients with colorectal cancer. Colorectal Dis 2012; 14: e375-e377

9 Lee SK, Kim TI, Shin SJ et al. Impact of prior abdominal or pelvic surgery on colonoscopy outcomes. J Clin Gastroenterol 2006; 40: 711 - 716

10 Garrett KA, Church J. History of hysterectomy: a significant problem for colonoscopists that is not present in patients who have had sigmoid colectomy. Dis Colon Rectum 2010; 53: 1055-1060

11 Kisloff B, Peele PB, Sharam R et al. Quality of patient referral information for open-access endoscopic procedures. Gastrointest Endosc 2006; 64: $565-569$ 